\title{
Ontogenetic differences in localization of glutamine transporter ApGLNT1 in the pea aphid demonstrate that mechanisms of host/ symbiont integration are not similar in the maternal versus embryonic bacteriome
}

\author{
Hsiao-ling Lu'1,2,3, Daniel R. G. Price ${ }^{1}$, Athula Wikramanayake', Chun-che Chang ${ }^{2,3}$ and Alex C. C. Wilson ${ }^{1 *}$
}

\begin{abstract}
Background: Obligate intracellular symbionts of insects are metabolically and developmentally integrated with their hosts. Typically, reproduction fails in many insect nutritional endosymbioses when host insects are cured of their bacterial symbionts, and yet remarkably little is known about the processes that developmentally integrate host and symbiont. Here in the best studied insect obligate intracellular symbiosis, that of the pea aphid, Acyrthosiphon pisum, with the gammaproteobacterium Buchnera aphidicola, we tracked the expression and localization of amino acid transporter ApGLNT1 gene products during asexual embryogenesis. Recently being characterized as a glutamine transporter, ApGLNT1 has been proposed to be a key regulator of amino acid biosynthesis in A. pisum bacteriocytes. To determine when this important mediator of the symbiosis becomes expressed in aphid embryonic bacteriocytes, we applied whole-mount in situ hybridization and fluorescent immunostaining with a specific anti-ApGLNT1 antibody to detect the temporal and spatial expression of ApGLNT1 gene products during asexual embryogenesis.

Results: During embryogenesis, ApGLNT1 mRNA and protein localize to the follicular epithelium that surrounds parthenogenetic viviparous embryos, where we speculate that it functions to supply developing embryos with glutamine from maternal hemolymph. Unexpectedly, in the embryonic bacteriome ApGLNT1 protein does not localize to the membrane of bacteriocytes, a pattern that leads us to conclude that the regulation of amino acid metabolism in the embryonic bacteriome mechanistically differs from that in the maternal bacteriome. Paralleling our earlier report of punctate cytoplasmic localization of ApGLNT1 in maternal bacteriocytes, we find ApGLNT1 protein localizing as cytoplasmic puncta throughout development in association with Buchnera.

Conclusions: Our work that documents ontogenetic shifts in the localization of ApGLNT1 protein in the host bacteriome demonstrates that maternal and embryonic bacteriomes are not equivalent. Significantly, the persistent punctate cytoplasmic localization of ApGLNT1 in association with Buchnera in embryos prior to bacteriocyte formation and later in both embryonic and maternal bacteriomes suggests that ApGLNT1 plays multiple roles in this symbiosis, roles that include amino acid transport and possibly nutrient sensing.
\end{abstract}

Keywords: Host/symbiont developmental integration, Coevolution, Symbiosis, Amino acid transport, Holobiont, Bacteriome

\footnotetext{
*Correspondence: acwilson@bio.miami.edu

${ }^{1}$ Department of Biology, University of Miami, Coral Gables, FL 33146, USA

Full list of author information is available at the end of the article
} 


\section{Background}

Vertically transmitted symbionts are developmentally and metabolically integrated with their hosts to generate the holobiont (host + symbiotic microorganism) [1] In most systems, remarkably little is known about the mechanisms mediating holobiont integration. Here we address this knowledge deficit by studying integration of the pea aphid, Acyrthosiphon pisum, and its endosymbiont, Buchnera aphidicola, during parthenogenetic viviparous embryogenesis using $A$. pisum glutamine transporter, ApGLNT1 [2].

Buchnera are the maternally inherited obligately intracellular nutritional symbionts of almost all extant aphids [3, 4]. In adult females, Buchnera are contained in a maternal bacteriome, which is an organ-like structure that comprises an aggregation of bacteriocytes and sheath cells. Each bacteriocyte houses thousands of individual Buchnera enveloped in host-derived symbiosomal membranes, while sheath cells that are located at the periphery of bacteriocytes and sometimes contain secondary bacterial symbionts do not contain Buchnera [5-7]. The transovarial inheritance and developmental integration of Buchnera can be divided into three phases: transmission, cellularization, and maturation. Occurring early in the development of blastoderm embryos, transmission involves exocytosis of Buchnera from maternal bacteriocytes-a process that results in release of Buchnera from maternal bacteriocytes and loss of the symbiosomal membrane. Following release, naked Buchnera move through the maternal extracellular space via cytoplasmic extensions that extend from maternal bacteriocytes to the inner space of the blastula [8]. Prior to gastrulation, Buchnera invade blastula embryos between the posterior enlarged follicle cells [9] resulting in Buchnera cells reacquiring their host-derived symbiosomal membrane [8]. After invasion, Buchnera cells aggregate in the posterior egg chamber with uncellularized host nuclei $[9,10]$. Cellularization-the second phase of developmental integration-follows embryo gastrulation when the Buchnera population compartmentalizes into individual bacteriocytes and proliferates [10]. Maturationthe final phase of developmental integration-occurs during late embryogenesis (stages 16-19) after katatrepsis (also known as "embryo flip", the second event of the blastokinesis that is peculiar to hemimetabolous insects). During maturation, uninucleate bacteriocytes and the intervening sheath cells located around the bacteriocytes form the dorsally localized bacteriome [8-10].

Buchnera, like other obligate intracellular symbionts of insects that feed on plant sap, provide their hosts with essential amino acids that are found only at very low concentrations in plant sap [11]. Amino acid biosynthesis in the A. pisum/Buchnera holobiont occurs in bacteriocytes.
Transport of amino acids from aphid hemolymph into bacteriocytes across the symbiosomal membrane and the inner and outer membranes of Buchnera and then back out to aphid hemolymph is central to symbiotic function. Recently, one amino acid transporter, ApGLNT1, has been proposed to regulate amino acid biosynthesis in bacteriocytes, thus ensuring that amino acid supply meets host demand [2].

Amino acid transporter ApGLNT1 is part of an arthropod expanded clade of eukaryotic-specific amino acid/ auxin permease (AAAP) family transporters (Transporter Classification \# 2.A.18) and closely related to the mammalian solute carrier 36 (SLC36) family [12]. Notably, the predicted membrane topology and protondependent uptake characteristics of ApGLNT1 are similar to those of the mammalian SLC36 family [13, 14]. Being highly expressed in A. pisum gut and bacteriocyte tissue, ApGLNT1 has remarkably narrow substrate selectivity with high glutamine and low arginine transport function $[2,12,13]$. While the arginine transport capacity of ApGLNT1 is low, its arginine binding affinity is high and, thus, arginine functions as a competitive inhibitor of glutamine transport [2]. Coupling the transport capacity of ApGLNT1 with its localization to the membrane of adult bacteriocytes led Price et al. [2] to propose that ApGLNT1 is the key regulator of amino acid biosynthesis in the A. pisum/Buchnera holobiont.

Amino acid transporters play an important role in amino acid transport at the symbiotic interface in all biological systems that include an obligate intracellular symbiont. One group of insects that critically depend on vertically transmitted, obligate intracellular symbionts includes plant sap-feeding insects of the suborder Sternorrhyncha. Remarkably, in earlier work we have found that nutrient amino acid transporter gene families are expanded in Sternorrhyncha insects that include the whitefly Bemisia tabaci, the potato psyllid Bactericera cockerelli, the citrus mealybug Planococcus citri, and the pea aphid Acyrthosiphon pisum $[15,16]$. In all these insects, a subset of amino acid transporter paralogs shows patterns of bacteriocyte-biased gene expression suggesting that neofunctionalization or subfunctionalization of duplicated paralogs facilitates host/symbiont metabolic integration [12, 15]. While the genomic basis of host/symbiont metabolic integration is increasingly well understood [17], the developmental basis of host/symbiont integration has been less studied. That said, recent work in the hemipteran insect Nysius plebeius has started to reveal the molecular and developmental mechanisms that drive bacteriocyte differentiation [18]. Here we study the developmental integration of the nutritional symbiont Buchnera by extending the work of Price et al. [2] to investigate the expression and localization of ApGLNT1 
gene products through viviparous development in the pea aphid. We show that RNA expression and protein localization of ApGLNT1 are different in the maternal versus embryonic bacteriome leading us to conclude that host/symbiont integration and regulation are not ontogenetically constant.

\section{Methods \\ Aphids}

A. pisum line LSR1 [19] was maintained on Vicia faba and incubated at $20^{\circ} \mathrm{C}$ under a 16-h light/8-h dark cycle. Oocytes, embryos, and bacteriocytes were dissected for whole-mount RNA in situ hybridization and fluorescent immunostaining from wingless adult females in phosphate-buffered saline (PBS; $10 \mathrm{mM}$ phosphate buffer, $154 \mathrm{mM} \mathrm{NaCl}, \mathrm{pH}$ 7.4; Sigma-Aldrich). Oogenesis and embryogenesis staging was according to Miura et al. [9] with germ cell locations according to Chang et al. [20].

\section{Validation of Anti-ApGLNT1 antibody against ApGLNT1 protein}

Here we use the monospecific anti-ApGLNT1 antibody purified from rabbit sera as described by Price et al. [2]. This antibody was raised against amino acids 28-40 of ApGLNT1 (LDNNKRGSIRTDV). Earlier validation by Price et al. [2] of this antibody included preadsorbed controls run in parallel with all experiments. Here we further validate the anti-ApGLNT1 antibody by Western blot. Briefly, a full-length coding sequence for ApGLNT1 [GenBank: NM_001246261.1] was amplified from whole adult female $A$. pisum cDNA using Phusion proof-reading polymerase (Finnzymes). The primers contained a $5^{\prime}$ optimized Kozak initiation sequence for efficient translation in yeast [21], a $5^{\prime}$ NotI site, and a $3^{\prime}$ BamHI site. The forward primer sequence is $5^{\prime}$-AAGCGGCCGCAT AATGGCGCATCATT- $3^{\prime}$ and the reverse primer sequence is $5^{\prime}$-TTGGATCCTCACTGATTGAACGTT $\mathrm{AC}-3^{\prime}$; restriction enzyme sites are shown in bold. The amplified ApGLNT1 coding sequence was digested with NotI and BamHI and cloned into the respective sites of the yeast shuttle vector pDR195 [22]. The ApGLNT1 expression construct was fully sequenced and used to transform Saccharomyces cerevisiae strain 22 $\Delta 8$ AA [23] by the lithium acetate/PEG method [24]. Transformants were selected on synthetic complete (SC) media, pH 5.6 $(0.17 \%$ yeast nitrogen base, $2 \%$ maltose, $1 \%$ agar supplemented with uracil drop-out mix) at $30{ }^{\circ} \mathrm{C}$ for $3-4$ days. Controls were run in parallel and consisted of cells transformed with empty pDR195 expression vector (negative control).

Total membrane fractions were isolated from $22 \Delta 8 \mathrm{AA}$ cells as described by Sauer and Stolz [25]. Membrane proteins were resolved by SDS-PAGE on a $12.5 \%$ polyacrylamide gel containing $0.1 \%$ SDS in a discontinuous pH system [26]. Separated proteins were transferred to nitrocellulose membranes by electroblotting in Bjerrun and Schafer-Nielsen buffer with SDS (48 mM Tris, $39 \mathrm{mM}$ glycine, $20 \%$ methanol, $0.0375 \%$ SDS, pH 8.3). Transferred proteins were probed with rabbit antiApGLNT1 antibody [2] at 1:1000 dilution, followed by secondary IRDye $800 \mathrm{CW}$ Goat anti-rabbit IgG $(\mathrm{H}+\mathrm{L})$ (LI-COR) at 1:10,000 dilution. Bound antibodies were visualized using a LI-COR Odyssey infrared imaging system model 9120.

Western blot analysis revealed that the anti-ApGLNT1 polyclonal antibody recognizes a single major band of recombinant ApGLNT1 protein that was extracted from the membrane fractions of the yeast $S$. cerevisiae expressing ApGLNT1 (Additional file 1: Figure S1). These data validate that the anti-ApGLNT1 antibody is specific to endogenous ApGLNT1 as described by Price et al. [2].

\section{Whole-mount RNA in situ hybridization}

Riboprobes were prepared from a $509 \mathrm{bp}$ fragment of the ApGLNT1 coding sequence in a region present in all four ApGLNT1 alternative splice variants present in the NCBI database, i.e., 524-1032 of NM_001246261, 1182-1690 of XM_008184610, 1176-1684 of XM_008184611, and 543-1051 of XM_008184612. ApGLNT1 was amplified from plasmids containing full-length ApGLNT1 cDNA [2] using forward 5'-CCTTCCAAAAATTTTCCGGT-3' and reverse $5^{\prime}$-GAGGAAGCCAAACATTCCAA- $3^{\prime}$ primers. Amplification conditions consisted of an initial denaturation at $94{ }^{\circ} \mathrm{C}$ for $30 \mathrm{~s}$, followed by 35 cycles at $94{ }^{\circ} \mathrm{C}$ for $10 \mathrm{~s}, 50{ }^{\circ} \mathrm{C}$ for $30 \mathrm{~s}, 72{ }^{\circ} \mathrm{C}$ for $1 \mathrm{~min}$, and a final extension at $72{ }^{\circ} \mathrm{C}$ for $5 \mathrm{~min}$. Amplicons were subcloned into pGEM-T vector (Promega). Finally, DIG-labeled sense and antisense $A p G L N T 1$ riboprobes were in vitro transcribed from linearized plasmids containing verified ApGLNT1 sequences using SP6 RNA polymerase (New England Biolabs) and T7 RNA polymerase (New England Biolabs), respectively.

Bacteriocytes and ovaries containing developing oocytes and embryos were fixed in $3.8 \%$ formaldehyde (VWR) in PBS at $4{ }^{\circ} \mathrm{C}$ overnight. The working concentration of each probe, including sense and anti-sense strands, was $3.0 \mathrm{ng} / \mu \mathrm{l}$. Other steps follow the protocol of Chang et al. [27]. Probe hybridization was performed at $68{ }^{\circ} \mathrm{C}$ and nitroblue tetrazolium (NBT)/5-bromo-4chloro-3-indolyl phosphate (BCIP) (Roche) was applied as the substrate for signal development. The substrate reaction was terminated concurrently for experimental and control treatments based on the relative intensity of signal in the follicle cells in the anti-sense riboprobe (experimental) and sense riboprobe (control) treatments. Following signal development, samples were mounted 
in $70 \%$ glycerol (Sigma-Aldrich) in PBS and photographed with a Zeiss Axiovert 200 microscope connected to a Zeiss AxioCam ICc1 camera. The experiment was performed five times for embryos and three times for bacteriocytes.

\section{Fluorescent immunostaining in oocytes and embryos}

Dissected ovaries that included developing oocytes and developing embryos were fixed in $4 \%$ paraformaldehyde (Thermo Scientific) in PBS for 20 min. Immunostaining followed the protocol of Chang et al. [28] but omitted the $\mathrm{H}_{2} \mathrm{O}_{2}$ treatment step. Ovaries were incubated in a 1:20 dilution of rabbit anti-ApGLNT1 polyclonal antibody [2] at $4{ }^{\circ} \mathrm{C}$ overnight, and the rabbit IgGs were detected with Alexa Fluor 633-conjugated goat anti-rabbit IgG $(\mathrm{H}+\mathrm{L})$ antibody (Invitrogen) at 1:500 dilution for 2-4 $\mathrm{h}$ at room temperature. Controls included a secondary antibody only negative control and an ApVasa1 (ApVas)positive control [29]. Nuclei and F-actin were stained with $2 \mu \mathrm{g} / \mathrm{ml}$ of $4^{\prime}, 6$-diamidino-2-phenylindole (DAPI) (Sigma-Aldrich) and $0.5 \mu \mathrm{g} / \mathrm{ml}$ of phalloidin-tetramethylrhodamine B isothiocyanate (phalloidin-TRITC) (Sigma-Aldrich) at room temperature for $1 \mathrm{~h}$. Samples were then mounted in $70 \%$ glycerol (Sigma-Aldrich) in PBS at $4{ }^{\circ} \mathrm{C}$ overnight. Images were acquired using a Leica TCS SP5 laser scanning confocal microscope in the University of Miami, Department of Biology Microscopy Core Facility. Control treatments were run in parallel. The experiment was performed five times.

\section{Results}

ApGLNT1 mRNA is expressed in the maternal follicular epithelium external to embryos and in sheath cells of the post-embryonic bacteriome

During early development (stages 0-4), ApGLNT1 transcripts are detected in the follicle cells between the germarium that is composed of nurse cells and presumptive oocytes and the first egg chamber (Fig. 1a; Additional file 2: Figure S2a, b). From stage 4 onward, ApGLNT1 mRNA is preferentially expressed in the follicular epithelium surrounding the egg chambers (Fig. 1b-f). Notably, we found that $A p G L N T 1$ is enriched in a region around the follicular cell nuclei in the follicular epithelium (Fig. 1d, f; Additional file 2: Figure S2c-i). Such epithelial localization of $A p G L N T 1$ transcripts continues through katatrepsis until the end of development (stage 19). While the most obvious and persistent expression of ApGLNT1 occurs in the follicular epithelium, we also detected expression of ApGLNT1 in the central syncytium concurrent with symbiont invasion (stage 7; Fig. 1b). However, we did not find ApGLNT1-specific signals in the location of Buchnera, nor did we find it in the embryonic head, or the embryonic alimentary canal.
In addition to examining $A p G L N T 1$ expression through development, we studied the subcellular localization of ApGLNT1 transcripts in the maternal bacteriome of nymphal and adult asexual females. In the maternal bacteriome of nymphal and adult asexual females, we detected ApGLNT1 transcripts in sheath cells but not within the cytoplasm of the bacteriocytes (Fig. 1g-j).

The patterns described above were repeatable across all five embryonic and all three bacteriocyte experimental replicates.

\section{ApGLNT1 protein is localized to the maternal follicular epithelium and embryonic sheath cells}

ApGLNT1 protein localization is spatially coincident with ApGLNT1 transcripts in the maternal follicular epithelium. We observed two layers of contiguous punctate signals: one along the outer edge of the maternal epithelium and the other surrounding the embryos (Fig. 2a-c, $\left.a^{\prime}-c^{\prime}\right)$. As embryos develop and grow the maternal epithelium flattens, so that by around stage 7 it is difficult to discern the double layer of ApGLNT1 signal using confocal imaging (Fig. 3a, a').

At stage 7, concomitant with Buchnera invasion weak punctate signals of ApGLNT1 can be detected in the bacterial region (arrow in Fig. 3a, a'). The intensity of these puncta increases during early gastrulation (Fig. 3b, b', c, c'). From stage 7 to stage 11, the punctate staining of ApGLNT1 is enriched in the region occupied by Buchnera (Fig. 3a-c, $\left.a^{\prime}-c^{\prime}\right)$. However, starting at stage 12 , during the process of bacteriocyte cellularization, ApGLNT1 puncta are enriched at the periphery of bacteriocytes, eventually localizing to what we interpret to be the interstitial region between bacteriocytes (in mature bacteriomes this interstitial region is occupied by sheath cells) (Fig. 4a, a', d, d'). As development proceeds through stages 12 and 13, the intensity of the ApGLNT1-positive signal in interstitial regions around bacteriocytes increases (Fig. 4b, b', e, e'). Notably, during germband formation (stage 13) and limb bud formation (stage 14), ApGLNT1-positive signals are always found in the nucleus and cytoplasm of putative sheath cells (arrow in Fig. 4c, c', f, $\mathrm{f}^{\prime}$, and for more detail see Additional file 3: Movie S1).

Following katatrepsis (stage 16 onward) (Fig. 5a, a', $\left.b, b^{\prime}\right)$, the bacteriome undergoes maturation [8]. During maturation, ApGLNT1-positive signals are found in the maternal follicular epithelium (double arrowhead in Fig. 5a, $\mathrm{a}^{\prime}$ ) and the sheath cell precursors (arrow in Fig. 5b, $b^{\prime}$ ). In addition, ApGLNT1 signal can be observed in the central nervous system (double arrow in Fig. 5a, a') and at limb/abdomen junctions (open arrow in Fig. 5a, a'). We are confident that all signals were generated by the anti-ApGLNT1 antibody because the negative controls were always almost completely devoid of signal (Fig. 5c, $\mathrm{c}^{\prime}$ ). 


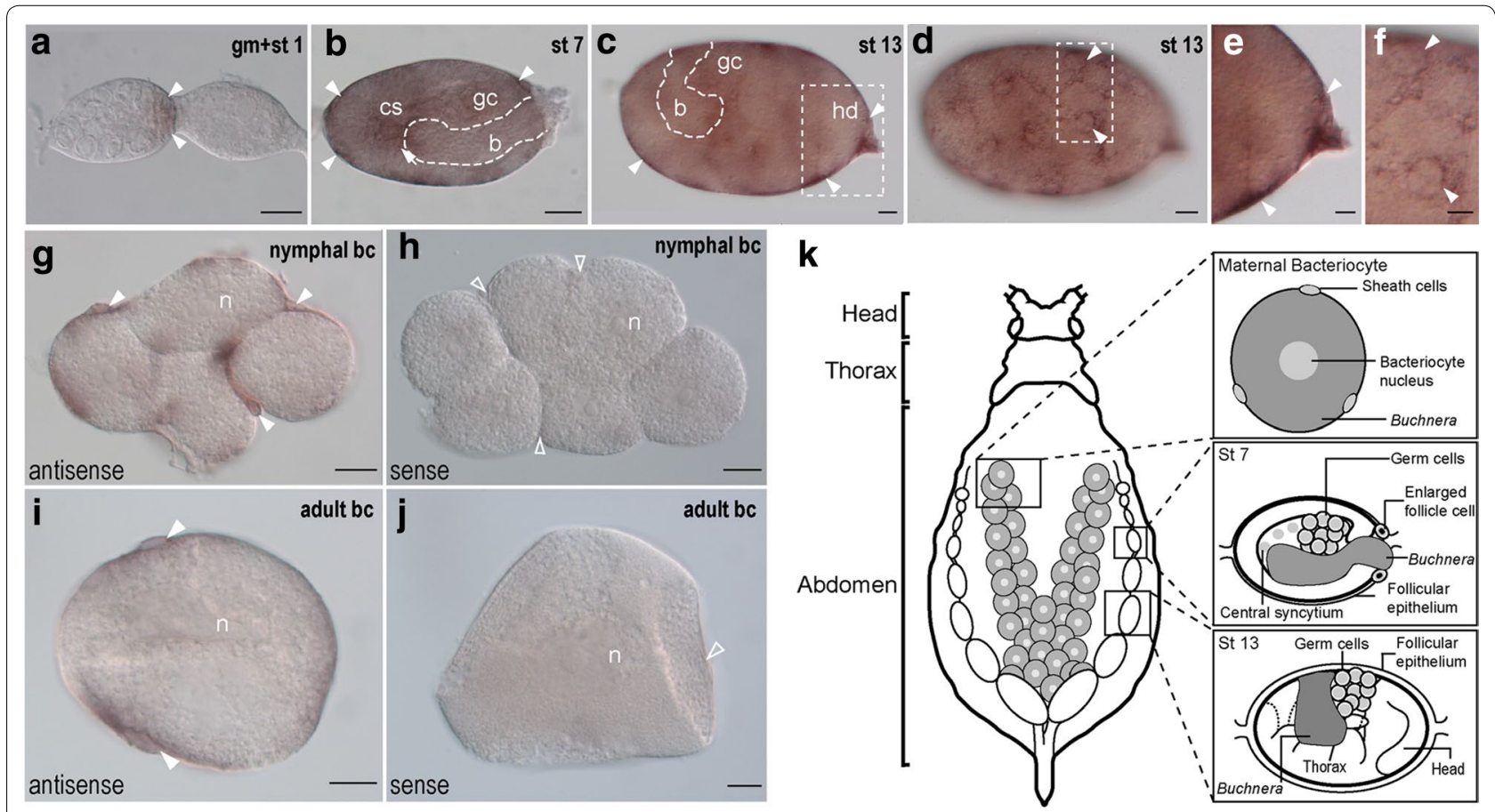

Fig. 1 Developmental expression of ApGLNT1 mRNA in the pea aphid. a-f Germaria and stage 1 oocyte (a), stage 7 (b) and stage 13 (c-f) embryos of the asexual pea aphid were hybridized with ApGLNT1 antisense riboprobes, and the transcripts were visualized with a color substrate NBT/BCIP. c and $\mathbf{d}$ are the same sample but at different focal planes. $\mathbf{e}$ and $\mathbf{f}$ are the insets of $\mathbf{c}$ and $\mathbf{d}$, respectively. The anterior of germaria is to the left. The head (hd) of germband is indicated in the posterior of the egg chamber to the right. The arrow indicates the antisense riboprobe-positive signal in the central syncytium (cs) and arrowheads indicate signals in the follicle cells in the periphery of egg chamber. $\mathbf{g}$-j Maternal bacteriome dissected from 24-48-h-old nymphs $(\mathbf{g}, \mathbf{h})$ and adult $(\mathbf{i}, \mathbf{j})$ hybridized with antisense and sense riboprobes. The expression of ApGLNT1 is indicated with closed arrowheads located in the sheath cells. The open arrowheads in the sense group indicate the regions where ApGLNT1 expression was detected when tissues were probed with anti-sense probes. $\mathbf{k}$ Illustration displaying presented bacteriocytes and developmental stages of embryos used for whole-mount in situ hybridization. Scale bars: $20 \mu \mathrm{m}$. b endosymbiotic bacteria Buchnera, bc bacteriocyte, cs central syncytium, $g c \mathrm{germ}$ cells, gm germarium, $h d$ head, $n$ bacteriocyte nucleus, st stage

The patterns described above were repeatable across all five replicate experiments. To validate immunostaining procedures and the efficiency of antibody penetration, all experiments included an ApVas-positive control. ApVas is a marker that specifically stains pea aphid primordial germ cells, and the localization of ApVas protein during development is well characterized (Fig. 5f) [29].

\section{Discussion}

\section{Protein localization patterns in the maternal} and embryonic bacteriomes are not equivalent

In the adult maternal $A$. pisum bacteriome, amino acid transporter ApGLNT1 localizes to the plasma membranes of bacteriocytes and sheath cells [2]. This mature localization of ApGLNT1 differs from the localization patterns we observed in the embryonic bacteriome. While we hypothesized that ApGLNT1 would localize to the plasma membrane of bacteriocytes and sheath cells following cellularization of the embryonic bacteriome (from stage 10 to the end of embryogenesis at stage 20), we only found transient co-localization of ApGLNT1 with F-actin on the inner face of the bacteriocyte plasma membrane at stage 14 (Fig. 4f, $\mathrm{f}^{\prime}$ ) and we did not observe a restricted signal surrounding single bacteriocytes in late-stage embryos (Fig. 5b, $\mathrm{b}^{\prime}$ ). The apparent transient nature of embryonic bacteriocytes enclosed with ApGLNT1-positive signal at stage 14 leaves us uncertain as to whether the membrane co-localization signals at stage 14 are signals from ApGLNT1 protein in the plasma membrane of bacteriocytes or of signal close to the boundary of bacteriocytes, an uncertainty that can be resolved in future work using higher resolution microscopy of samples co-stained with a plasma membranespecific marker.

In addition to localizing to the plasma membrane of bacteriocyte and sheath cells in the adult maternal bacteriome, ApGLNT1 localizes as puncta in the cytoplasm of maternal bacteriocytes [2]. During asexual embryogenesis, ApGLNT1 localizes as cytoplasmic puncta throughout development (see especially Figs. 2, 3, 4, and 5b, b'). While Price et al. [2] focused primarily on interpreting the membrane localization of ApGLNT1, the prominent 

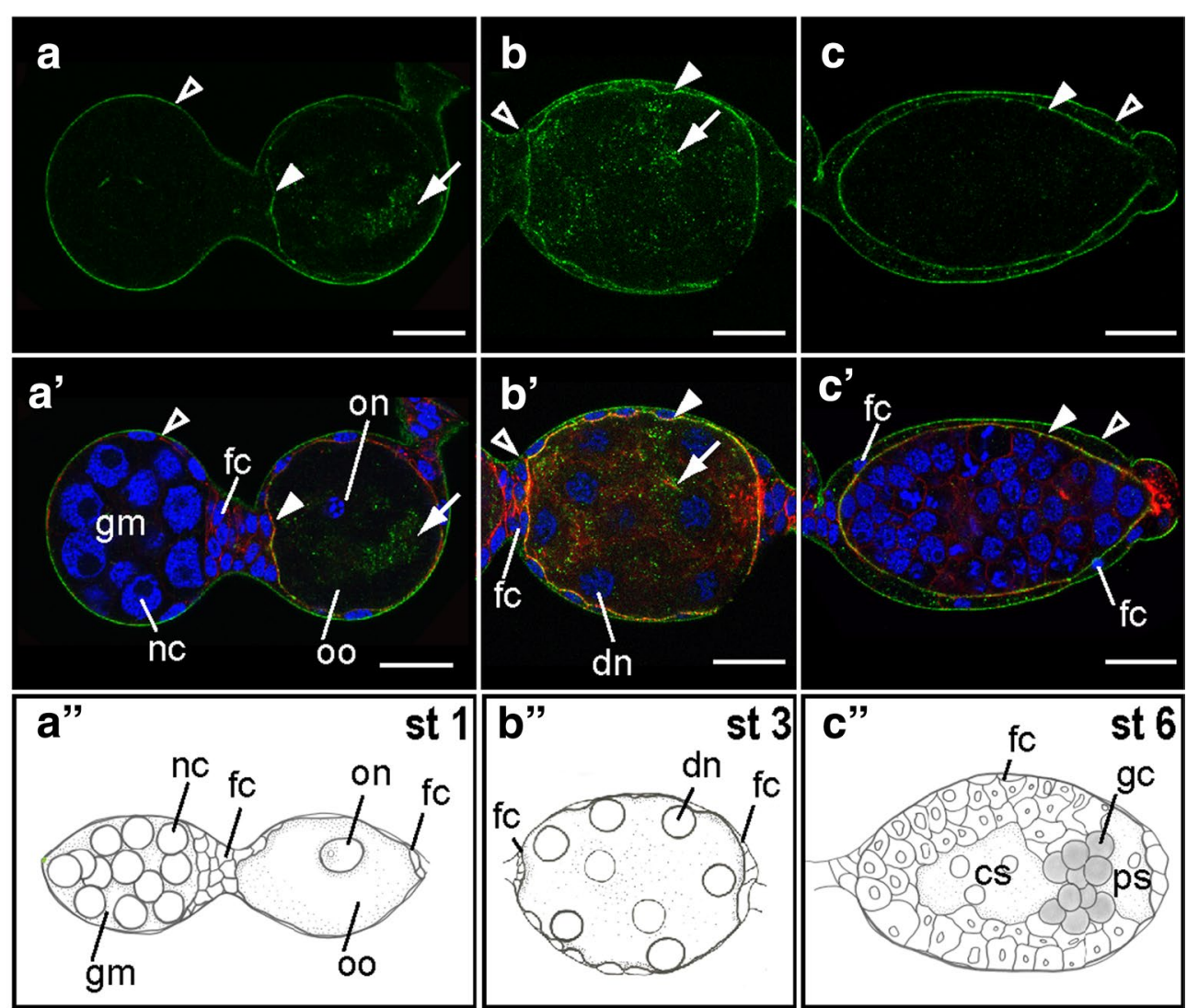

ApGLNT1
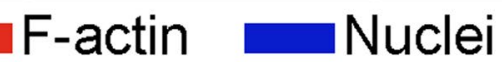

Fig. 2 Developmental expression of ApGLNT1 in early developing embryos before symbiont transmission. Staining with ApGLNT1 antibody was performed on dissected asexual embryos within the ovarioles. All embryos are displayed with anterior of the germaria to the left. Signals representing ApGLNT1 immunoactivity, F-actin to the cell cortex, and nuclei are marked with color keys at the bottom of the figure. Confocal images of a-c show ApGLNT1 antibody staining results. Confocal images of $\mathbf{a}^{\prime}-\mathbf{c}^{\prime}$ show merged results from ApGLNT1 antibody, nuclei, and F-actin staining. Illustrations of $\mathbf{a}^{\prime \prime}-\mathbf{c}^{\prime \prime}$ display the embryonic characteristics corresponding to the presented developmental stages. ApGLNT1 signal in the outer layer surrounding the whole ovarioles is indicated with open arrowheads, and the signal in the inner layer surrounding the developing oocyte and embryos is indicated with closed arrowhead. $\mathbf{a}, \mathbf{a}^{\prime}, \mathbf{a}^{\prime \prime}$ Germaria and developing oocyte (stage 1 of development). $\mathbf{a}, \mathbf{a}^{\prime}$ ApGLNT1 signals are detected in the membrane of follicular epithelium (closed and open arrowheads) and in the cytoplasm of the oocyte (arrow). $\mathbf{b}, \mathbf{b}^{\prime}, \mathbf{b}^{\prime \prime}$ Embryo undergoing synchronous, syncytial nuclear division with eight nuclei (stage 3 of development). $\mathbf{b}, \mathbf{b}^{\prime}$ ApGLNT1 signals are localized in the membrane of follicular epithelium (closed and open arrowheads) and in the cytoplasm of the syncytial embryos (arrow). $\mathbf{c}, \mathbf{c}^{\prime}, \mathbf{c}^{\prime \prime}$ Embryo with newly segregated germ cells (stage 6 of development). $\mathbf{c}, \mathbf{c}^{\prime}$ The membrane signals in the follicular epithelium remain detectable in the periphery of the embryos (closed and open arrowheads). Scale bars: $20 \mu \mathrm{m}$. cs central syncytium, dn dividing nuclei, fc follicle cells, gc germ cells, gm germarium, $n c$ nurse cells, on oocyte nucleus, oo oocyte, ps posterior syncytium, st stage

and persistent punctate patterns of cytoplasmic staining in embryos during asexual embryogenesis and in maternal bacteriocytes are remarkable and are interpreted later in this discussion where we suggest that cytoplasmic ApGLNT1 plays a role in nutrient sensing.

In the same way that localization of ApGLNT1 differs in embryonic and maternal bacteriocytes, ApGLNT1 localization differs between embryonic and maternal sheath cells. ApGLNT1 localizes to the plasma membrane of maternal sheath cells, and in contrast, in embryonic sheath cells it localizes as cytoplasmic puncta. Furthermore, the expression of ApGLNT1 transcript differs between embryonic and maternal sheath cells. ApGLNT1 mRNA is detected in maternal sheath cells in both first instar nymphs and adults (Fig. 1g, i) [30]; in contrast, we did not detect ApGLNT1 mRNA in embryonic sheath cells (Additional file 2: Figure S2g, h).

Our finding that ApGLNT1 expression and localization differs between maternal and embryonic bacteriomes demonstrates that bacteriome function is not ontogenetically constant, a pattern consistent with two other recent studies that also demonstrate differences in RNA expression and protein localization in embryonic versus maternal bacteriomes [31, 32]. This lack of equivalency between 

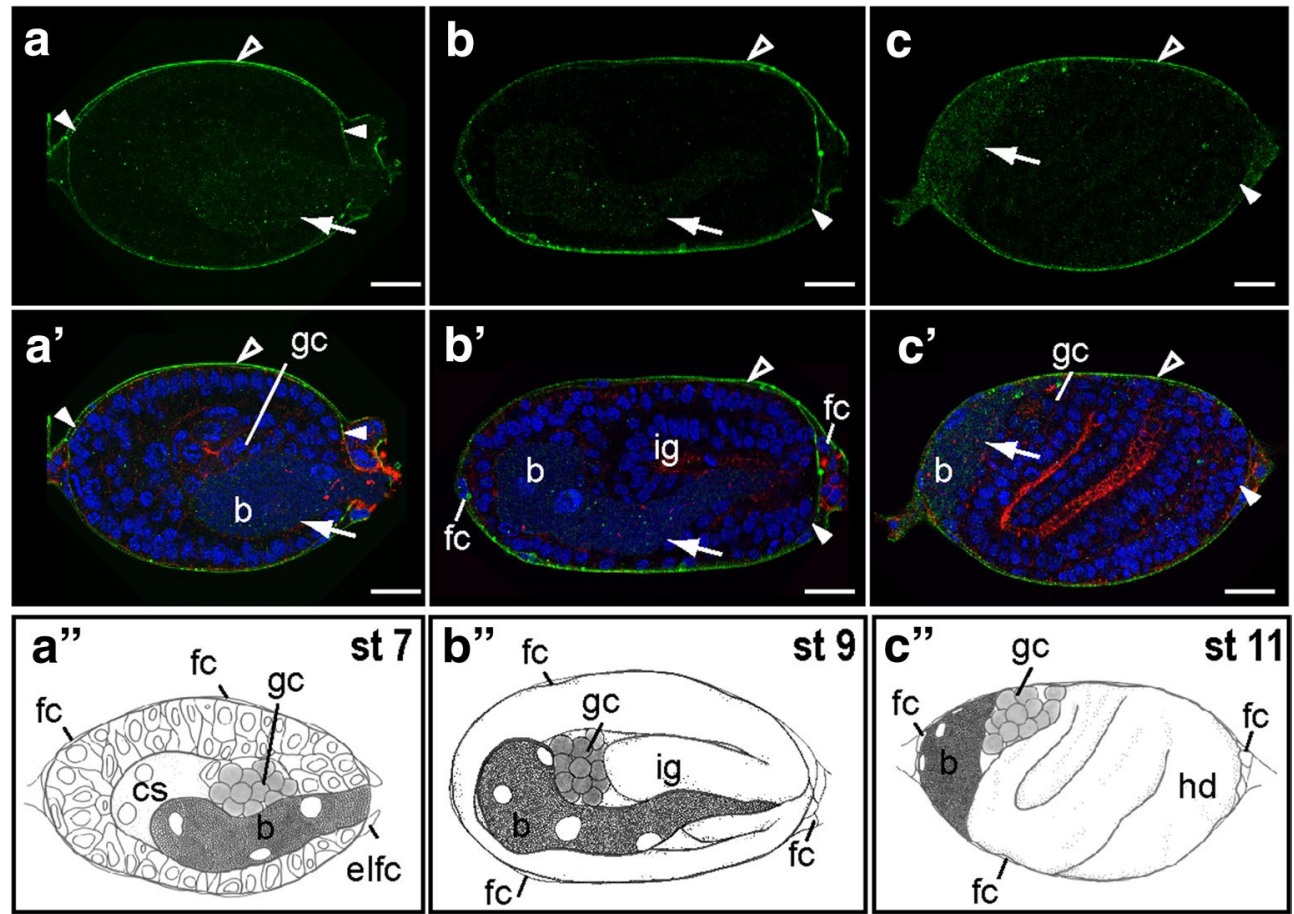

ApGLNT1

F-actin

Nuclei

Fig. 3 Developmental expression of ApGLNT1 in the embryos when the maternal symbiont undergoes transmission. Signals representing ApGLNT1 immunoactivity, F-actin to the cell cortex, and nuclei are marked with color keys at the bottom of the figure. All embryos are displayed with anterior of the germaria to the left. Confocal images of a-c show ApGLNT1 antibody staining results. Confocal images of $\mathbf{a}^{\prime}-\mathbf{c}^{\prime}$ show merged results from ApGLNT1 antibody, nuclei, and F-actin staining. Illustrations of $\mathbf{a}^{\prime \prime}-\mathbf{c}^{\prime \prime}$ display the embryonic characteristics corresponding to the presented developmental stages. ApGLNT1 signal in the outer layer surrounding the whole ovarioles is indicated with open arrowhead, and signal in the inner layer surrounding the developing embryos is indicated with closed arrowhead. The punctate signal of ApGLNT1 localized in the endosymbiotic bacteria region is indicated with an arrow. $\mathbf{a}, \mathbf{a}^{\prime}, \mathbf{a}^{\prime \prime}$ Embryo incorporating the maternal endosymbiotic bacteria (stage 7 of development). $\mathbf{a}, \mathbf{a}^{\prime}$ ApGLNT1 signals are detected in the membranes of the follicular epithelium (closed and open arrowheads) and in the invading endosymbiotic bacteria (arrow). $\mathbf{b}, \mathbf{b}^{\prime}, \mathbf{b}^{\prime \prime}$ Invaginating embryo (stage 9 of development). $\mathbf{b}, \mathbf{b}^{\prime}$ Similar to stage 7 embryo, ApGLNT1 signals are localized in the membranes of follicular epithelium (closed and open arrowheads) and in the invading endosymbiotic bacteria (arrow). $\mathbf{c}_{1} \mathbf{c}^{\prime}, \mathbf{c}^{\prime \prime}$ S-shaped embryo (stage 11 of development). $\mathbf{c}, \mathbf{c}^{\prime}$ The signal of follicular epithelium remains detectable in the periphery of the embryos (closed and open arrowheads) and punctate signals in the region of endosymbiotic bacteria become obvious (arrow). Scale bars: $20 \mu \mathrm{m}$. b endosymbiotic bacteria Buchnera, cs central syncytium, elfc enlarged follicle cells, fc follicle cells, gc germ cells, hd head, ig invaginating germband, st stage

embryonic and maternal bacteriomes raises intriguing questions about the nutritional contributions of Buchnera contained within embryonic bacteriomes during embryogenesis. Which bacteriome(s), the maternal and/ or the embryonic, support the extraordinary reproductive capacity of viviparous aphids? Functional characterization and developmental localization of transporters that facilitate transport of Buchnera synthesized amino acids and vitamins will facilitate addressing this long-standing question.

\section{Transovarial glutamine transportation may be mediated by ApGLNT1}

There are two membrane barriers that separate maternal hemolymph from developing embryos in asexual viviparous aphids: the follicular epithelium and the embryonic epithelium [33]. The follicular epithelium is the continuous monolayer of epithelial cells that surrounds the germarium and continues along the whole length of the ovariole [9, 34-37]. The embryonic epithelium envelops individual embryos. We interpret the double layer of ApGLNT1 antibody-positive signal to be localized to the outside membrane of the maternal follicular epithelium and the apical membrane of the embryonic epithelium (Figs. 2, 3, 4, 5). As embryos grow within an ovariole, the follicular epithelium stretches so that after stage 7 a double layer of ApGLNT1 signal is difficult to discern. Localization of ApGLNT1 in membranes of two of the cellular barriers that separate maternal hemolymph from embryonic hemolymph indicates that 


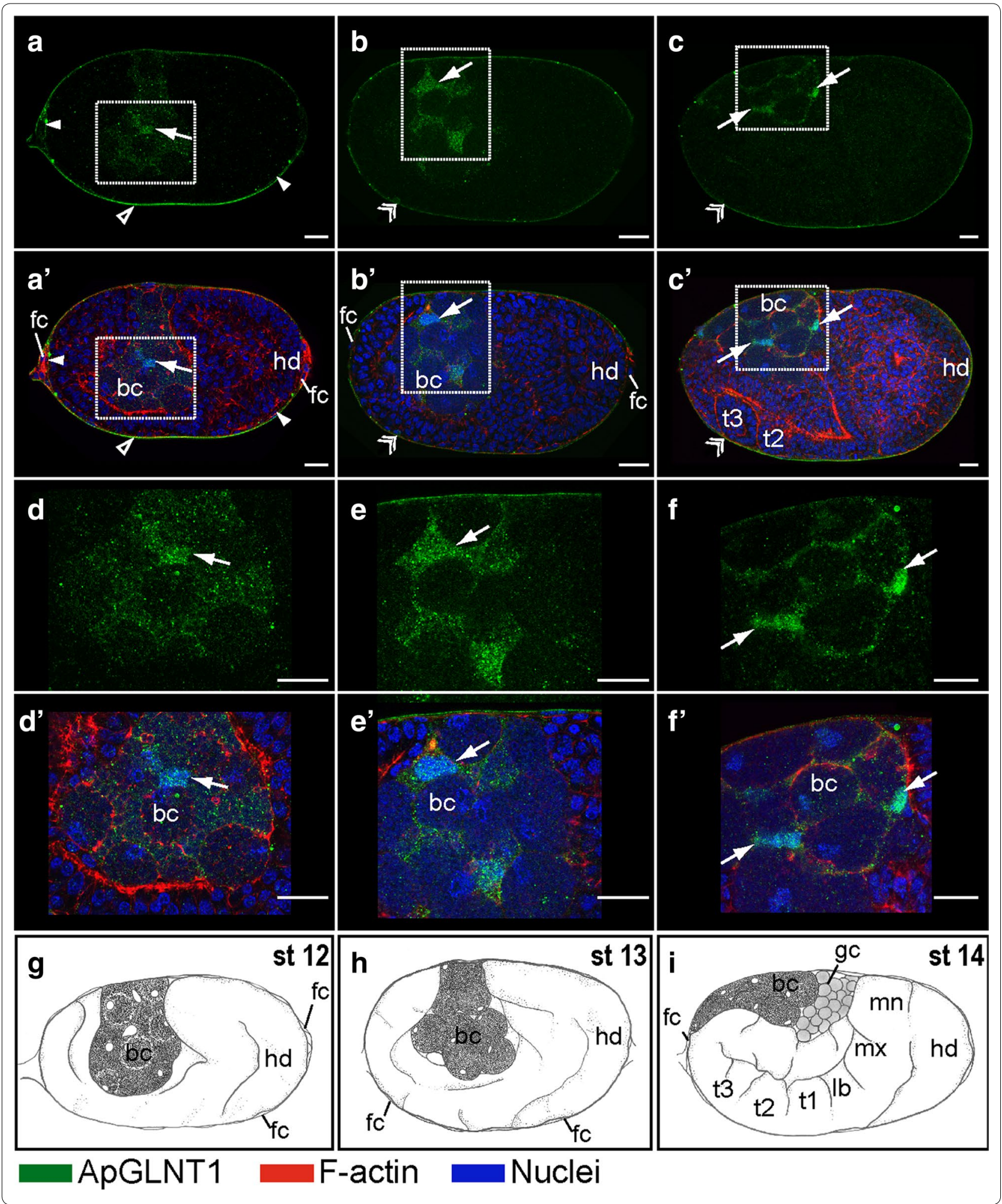

glutamine may be transported from maternal hemolymph to developing oocytes and embryos throughout asexual development.
The nutrient provisioning roles of maternal and embryonic bacteriomes during development are far from resolved [38]. Unlike most insects where maturation of 
(See figure on previous page.)

Fig. 4 Developmental expression of ApGLNT1 in the embryos when bacteriocyte undergoes cellularization. Signals representing ApGLNT1 immunoactivity, F-actin to the cell cortex, and nuclei are marked with color keys at the bottom of the figure. All embryos are displayed with anterior of the germaria to the left. Confocal images of $\mathbf{a}-\mathbf{c} ; \mathbf{d}-\mathbf{f}$ show ApGLNT1 antibody staining results. Confocal images of $\mathbf{a}^{\prime}-\mathbf{c}^{\prime} ; \mathbf{d}^{\prime}-\mathbf{f}^{\prime}$ show merged results from ApGLNT1 antibody, nuclei, and F-actin staining. $\mathbf{d}-\mathbf{f}$ and $\mathbf{d}^{\prime}-\mathbf{f}^{\prime}$ are the insets shown in $\mathbf{a}-\mathbf{c}$ and $\mathbf{a}^{\prime}-\mathbf{c}^{\prime}$, respectively. Illustrations of $\mathbf{g - i}$ display the embryonic characteristics corresponding to the presented developmental stages. ApGLNT1 signal in the outer layer surrounding the whole ovarioles is indicated with an open arrowhead, the inner layer surrounding the embryos is indicated with a closed arrowhead, and when two layer signals are indiscernible indicated with double arrowheads. ApGLNT1 signal located within the bacteria region is indicated with arrows. $\mathbf{a}, \mathbf{a}^{\prime}, \mathbf{d}, \mathbf{d}^{\prime}$, $\mathbf{g}$ Twisting embryo (stage 12 of development). $\mathbf{a}, \mathbf{a}^{\prime}, \mathbf{d}, \mathbf{d}^{\prime}$ ApGLNT1 signals are detected in the membranes of follicular epithelium (closed and open arrowheads) and in the periphery of cellularized bacteriocytes (arrow). $\mathbf{b}, \mathbf{b}^{\prime}, \mathbf{e}, \mathbf{e}^{\prime}, \mathbf{h}$ Embryo undergoing limb bud formation (stage 13 of development). $\mathbf{b}, \mathbf{b}^{\prime}, \mathbf{e}, \mathbf{e}^{\prime}$ Follicular epithelium signal can be detected (double arrowhead). ApGLNT1 signal in the periphery of the cellularized bacteriocytes becomes more condensed (arrow). $\mathbf{c}, \mathbf{c}^{\prime}, \mathbf{f}, \mathbf{f}^{\prime}, \mathbf{i}$ Extension of the germband (stage 14 of development). $\mathbf{c}, \mathbf{c}^{\prime}, \mathbf{f}, \mathbf{f}^{\prime}$ The follicular epithelium signal remains detectable in the periphery of the egg chamber (double arrowhead). ApGLNT1 signal in the periphery of the cellularized bacteriocytes becomes more restricted (arrows). Scale bars: $20 \mu \mathrm{m}$. bc bacteriocyte, fc follicle cells, gc germ cells, hd head, lb labial segment, $\mathrm{mn}$ mandible segment, $m \times$ maxilla segment, st stage, $t 1-t 3$ the three thoracic segments

oocytes occurs within the ovariole and embryos develop externally, asexual viviparous aphids have telotrophic ovarioles that contain nurse cells (at their terminal tip), developing oocytes and developing embryos that are all bathed in maternal hemolymph. During early development (before stage 4), asexual embryos are provisioned
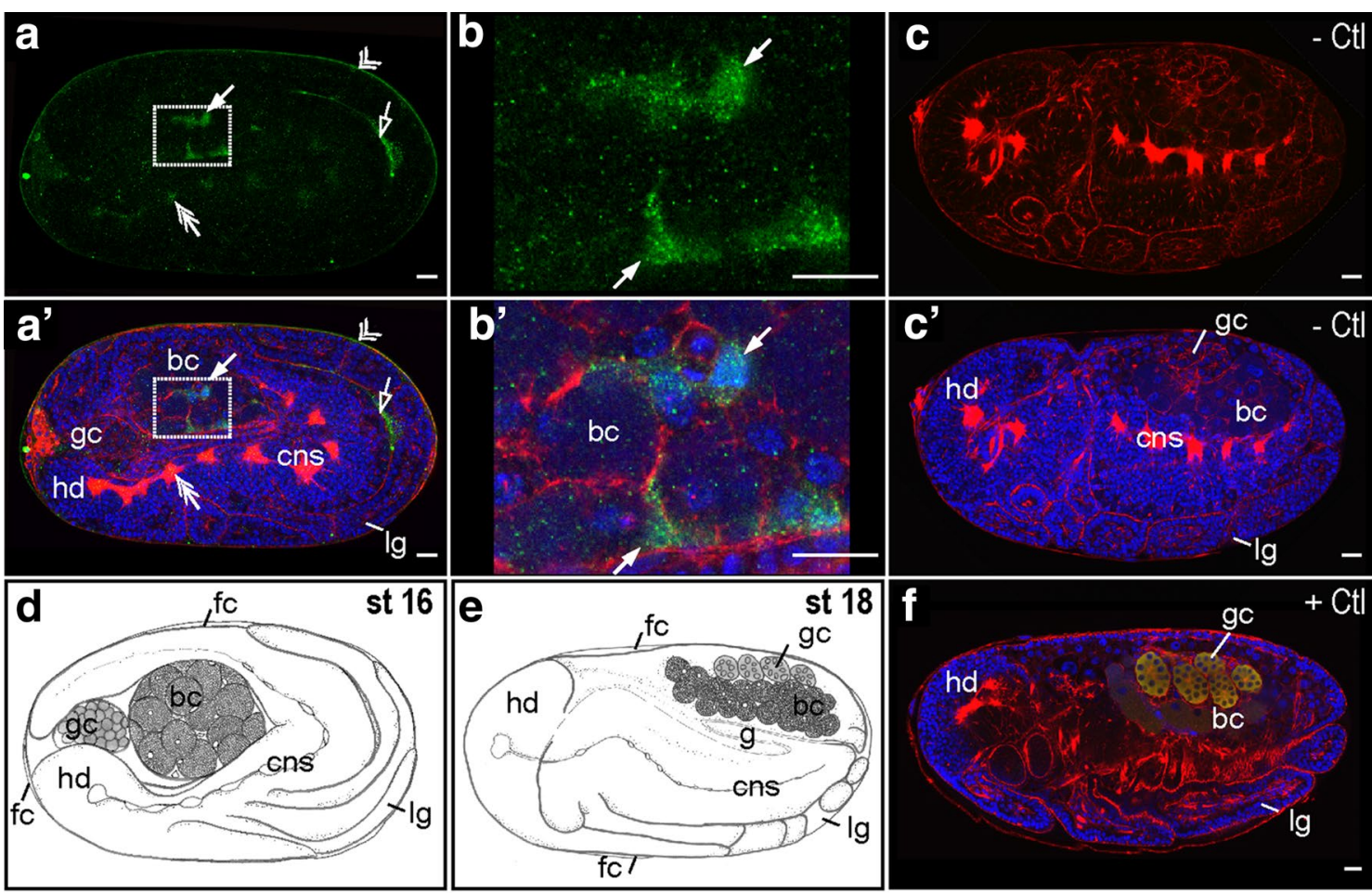

\section{ApGLNT1}

ApVas

F-actin

Nuclei

Fig. 5 Developmental expression of ApGLNT1 in post-katatrepsis embryos when bacteriome undergoes maturation. Signals representing ApGLNT1 and ApVas immunoactivity, F-actin, and nuclei are marked with color keys below the figure. All embryos are displayed with anterior of the germaria to the left. Confocal images of $\mathbf{a}$ and $\mathbf{b}$ show ApGLNT1 antibody staining results. $\mathbf{a}^{\prime}$ and $\mathbf{b}^{\prime}$ show merged results from ApGLNT1 antibody, nuclei, and F-actin staining. $\mathbf{b}$ and $\mathbf{b}^{\prime}$ are the insets of $\mathbf{a}$ and $\mathbf{a}^{\prime}$, respectively. $\mathbf{c}$ and $\mathbf{c}^{\prime}$ are the negative controls. Illustrations of $\mathbf{d}$ and $\mathbf{e}$ display the embryonic characteristics corresponding to the presented developmental stages. $\mathbf{f}$ ApVas-positive control of staining. $\mathbf{a}, \mathbf{a}^{\prime}, \mathbf{b}, \mathbf{b}^{\prime}$ Embryo after flip (stage 16). $\mathbf{a}, \mathbf{a}^{\prime}, \mathbf{b}, \mathbf{b}^{\prime}$ The ApGLNT1 signal can be detected in the follicular epithelium (double arrowhead), in the presumptive bacterial sheath cells (arrow) of the bacteriome, and in the central nervous system (double arrow). An ApGLNT1-positive signal can be detected in the space of germband and limb bud (open arrow). c, $\mathbf{c}^{\prime}$ Embryo undergoes retraction (stage 17). c Embryo stained with only secondary antibody and merged with the F-actin staining and $\mathbf{c}^{\prime}$ merged with nucleus staining. In the absence of anti-ApGLNT1 antibodies, no immunostaining signal is detected. $\mathbf{f}$ Stage 18 embryo stained with ApVas antibody as a positive control. Signal specifically appears in the germ cells. Scale bars: $20 \mu \mathrm{m}$. bc bacteriocyte, cns central nervous system, fc follicle cells, g gut, gc germ cells, hd head, Ig legs, st stage, - Ctl: negative control, + Ctl: positive control 


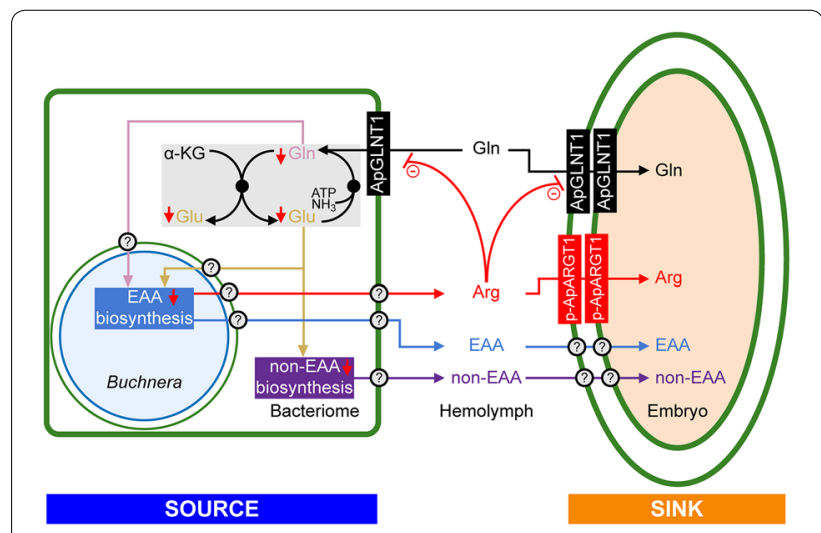

Fig. 6 Model for holobiont metabolism regulated by the nutritional demand of the asexual aphid reproductive system. The non-essential amino acid (non-EAA) glutamine (Gln) is transported from hemolymph to the bacteriome and developing embryos by transporter ApGLNT1. Glutamine and glutamate (Glu) are interconverted in the cytoplasm of the maternal bacteriome via the aphid genome-encoded GOGAT cycle (shaded gray) [51]. Glutamine and glutamate serve as metabolic precursors for amino acid biosynthesis in the holobiont [1]. We extend Price et al's [2] regulatory model of amino acid biosynthesis in the maternal bacteriome to include the nutrient demands of embryogenesis such that developing embryos within viviparous aphids act as sinks that consume essential and non-essential amino acids. Arginine (Arg) demand in the embryos will maintain amino acid biosynthesis in the maternal bacteriome. Thus, we propose the localization of an as yet unidentified arginine transporter, putative A. pisum Arginine Transporter 1, p-ApARGT1, on the outside membrane of the maternal follicular epithelium and the apical membrane of the embryonic epithelium. The membranes that ApGLNT1 has been shown to localize to are shown with a thick green line. These membranes include the bacteriome and follicular epithelial membranes. The symbiosomal membrane and Buchnera cell membrane are marked with a thin green line and thin blue line, respectively. Uncharacterized transporters are indicated by question marks

with nutrients by nurse cells via the nutritive cord that directly connects them to the germarium [39, 40]. After dissolution of the nutritive cord, embryos likely receive nutrients directly from the maternal hemolymph. However, following stage 7, when Buchnera symbionts invade developing embryos, it is possible that some nutrient provisioning may be compartmentalized within each embryo, with nutrient upgrading and recycling occurring in the embryonic bacteriome. Speculation aside, prior to this study, the role(s) of the maternal versus embryonic symbiosis in nutrient provisioning to mid- and late-stage embryos was undetermined. Here we speculate through localization of ApGLNT1 mRNA and protein (Figs. 1, $2,3,4,5)$ that glutamine is transported from maternal hemolymph to developing embryos under conditions of low hemolymph arginine availability throughout development (Fig. 6) [2, 13]. The fact that ApGLNT1 is not found in the membranes of embryonic bacteriocytes also suggests that the mechanisms regulating amino acid biosynthesis in embryonic bacteriocytes differ from those regulating amino acid biosynthesis in maternal bacteriomes [2].

\section{Cytoplasmic localization of ApGLNT1 suggests that this} amino acid transporter may play a role in nutrient sensing ApGLNT1 is part of the arthropod expansion of mammalian SLC36 family transporters and is related to the mammalian proton-dependent amino acid transporters (PAT1-4) [12, 13]. Notably, both mammalian and Drosophila cytosolic SLC36 transporters have been shown to function as intracellular amino acid sensors [41]. Among the metazoans, the major amino acid sensing signaling pathways are the target of rapamycin (TOR) pathway and the general amino acid control non-derepressible 2 (GCN2) pathway [42]. Amino acid transporters play important roles at the top and bottom of both pathways by monitoring both intracellular and extracellular amino acid abundances [43]. In particular, Drosophila SLC36 homologs pathetic and CG1139, and human SLC36A1 are essential mediators that activate the key factor of Target of Rapamycin Complex 1 (TORC1) kinase in the TOR signaling pathway [41]. Overexpression and mutation of SLC36 gene products affects cell growth through modulating TORC1 $[44,45]$. Work with both mammalian and Drosophila cytosolic SLC36 transporters suggests that ApGLNT1 may also play a role in nutrient sensing. Alternatively, cytoplasmic localization of ApGLNT1 may result from transporter sequestration in vesicular membranes for later mobilization to the plasma membranes of bacteriocyte and sheath cells, where ApGLNT1 can then function to transport glutamine. That said, the possibility that ApGLNT1 plays both amino acid transport and nutrient sensing roles at the A. pisum/Buchnera symbiotic interface is intriguing. Resolution of this intrigue will require further experimental work that is outside the scope of the present study.

The intriguing possibility that ApGLNT1 functions during host development both as a nutrient sensor and glutamine transporter raises questions about how symbiotic function changes during development. Recent work comparing protein expression patterns in embryonic and maternal bacteriocytes revealed that protein expression differs between the embryonic and post-embryonic developmental stages, especially with respect to proteins related to the biosynthesis of essential amino acids [31], a result that is consistent with other studies that also found ontogenetic shifts in gene expression of genes central to the aphid-Buchnera nutritional association [32, 46, 47]. Changes in symbiotic function in response to nutrient demand or environmental cues are also integrated into host developmental programs in the human-gut 
microbiome [48], the squid-vibrio symbiosis [49], and the symbiosis of the salamander Ambystoma maculatum with its algal symboint, Oophila amblystomatis [50]. Indepth investigation of the cellular function of symbiontbiased genes during host development may facilitate elucidation of how symbiotic partners are integrated to generate the holobiont or, more particularly, how vertically transmitted symbionts are integrated into the developmental programs of their hosts.

\section{Conclusions}

That ApGLNT1 localizes to the A. pisum follicular epithelium suggests transport of glutamine from maternal hemolymph to embryos throughout asexual embryogenesis. Consistent with previous work, we show that the maternal and embryonic bacteriomes are not equivalent $[31,32,46]$ and venture that the extraordinary reproductive capacity of asexually reproducing aphids is largely supported by the maternal symbiosis. Remarkably, our work suggests that maternal bacteriocytes do not synthesize ApGLNT1 transcripts, but rather are provisioned with ApGLNT1 by neighboring sheath cells. Finally, the subcellular localization of ApGLNT1 in maternal and embryonic sheath and bacteriocyte cells informs our proposal that in addition to its role in nutrient transport, ApGLNT1 likely plays an important role in nutrient sensing at the A. pisum/Buchnera symbiotic interface.

\section{Additional files}

Additional file 1: Figure S1. Western blot analysis of recombinant $A$. pisum ApGLNT1 in yeast cell membranes. Western blot analysis of total membranes (20 mg membrane protein/lane) from S. cerevisiae strain 22 $\triangle 8 \mathrm{AA}$ [23] transformed with empty pDR195 expression vector (control, lane 1) and cells expressing ApGLNT1 (ApGLNT1, lane 2). Yeast membrane proteins were separated on a $12.5 \%$ polyacrylamide gel, transferred to nitrocellulose and probed with primary anti-ApGLNT1 antibody and a fluorescently labeled secondary antibody. Arrow indicates presence of an ApGLNT1 antibody immunoreactive band, which is only present in cells expressing ApGLNT1 (ApGLNT1, lane 2). The calculated relative molecular mass $\left(M_{\mathrm{r}}\right)$ of ApGLNT1 is $55.1 \mathrm{kDa}$, but as analyzed by SDS-PAGE the protein has an apparent $M_{\mathrm{r}}$ of $42 \mathrm{kDa}$. The anomalous migration of ApGLNT1 on SDS-PAGE gels is a due to the highly hydrophobic nature of the protein, and is consistent with other hydrophobic transmembrane transporters [52].

Additional file 2: Figure S2. Transcripts of ApGLNT1 localized to the maternal follicular epithelium in the asexual embryos. Dissected ovarioles were hybridized with antisense $(a-i)$ and sense riboprobes $(j-r)$. Anterior region of egg chambers is to the left and dorsal is to the top. After katatrepsis, the head of germband reverse the position to the anterior of the egg chambers $(i, r)$. Antisense positive signals mark with closed arrowheads in the follicular epithelium and arrow in the central syncytium. Scale bars: $20 \mu \mathrm{m}$. Abbreviation: b, endosymbiotic bacteria Buchnera; bc, bacteriocyte; gc, germ cells; gm, germarium; hd, head; st, stage.

Additional file 3: Movie S1. Cytoplasmic localization of ApGLNT1. Embryonic bacteriocytes movie in a stage 13 embryo constructed from serial confocal sections. Green, red, and blue signal are ApGLNT1-positive signal, F-actin and DNA.

\section{Abbreviations}

AAAP: amino acid/auxin permease family transporters; ApGLNT1: A. pisum glutamine transporter 1; BCIP: 5-bromo-4-chloro-3-indolyl phosphate; DAPI: 4',6-diamidino-2-phenylindole; DIG: digoxigenin; GCN2: general amino acid control non-derepressible 2; NBT: nitroblue tetrazolium; NCBI: National Center for Biotechnology Information; PATs: proton-dependent amino acid transporters; PBS: phosphate-buffered saline; phalloidin-TRITC: phalloidin-tetramethylrhodamine B isothiocyanate; SC: synthetic complete medium; SLC36: solute carrier family 36; SLC36A1: solute carrier family 36 member 1; TOR: target of rapamycin; TORC1: target of rapamycin complex 1 kinase.

\section{Authors' contributions}

ACCW conceived of the experiments. HLL, DRGP, AW, CCC, and ACCW designed the experiments. HLL and DRGP performed the experimental work and prepared the figures for publication. HLL, DRGP, and ACCW drafted the manuscript. All authors contributed to preparation of the submitted manuscript. All authors read and approved the final manuscript.

\section{Authors' information}

The confocal and in situ localization work reported here was completed by HLL while she was a fellow of the Taiwanese Ministry of Science and Technology Graduate Student Study Abroad Program in the laboratory of ACCW. HLL was a Ph.D. student in the laboratory of CCC studying developmental plasticity of germline development in the pea aphid. DRGP is a molecular biologist who uses functional genomic approaches to characterize the interaction between phloem-feeding insects and their obligate endosymbiotic bacteria. AW is a developmental biologist who studies the evolution of early embryonic pattern formation using marine invertebrate models. CCC is a developmental biologist who studies aphid oogenesis and embryogenesis. ACCW is an evolutionary biologist who studies the coevolution of plant sap-feeding insects and their obligate intracellular bacterial symbionts. Recent work in Wilson's group focuses on functional characterization of amino acid transporters in sternorrhynchan insects.

\section{Author details \\ 1 Department of Biology, University of Miami, Coral Gables, FL 33146, USA. ${ }^{2}$ Department of Entomology, College of Bioresources and Agriculture, National Taiwan University, Taipei, Taiwan. ${ }^{3}$ Research Center for Developmen- tal Biology and Regenerative Medicine, National Taiwan University, Taipei, Taiwan.}

\section{Acknowledgements}

We thank James Baker of the Confocal Microscopy Core Facility in the Department of Biology at the University of Miami, together with Lingyu Wang and Wei Wu, for technical support; and two anonymous reviewers whose comments helped improve the final manuscript. This work benefitted from useful discussions with James Baker, Honglin Feng, and Rebecca Duncan. HLL thanks G.W. Lin for the provision of pea aphid Vasa antibody. The financial support for the project came from a Ministry of Science and Technology Award 102-2917-I-002-006 (to HLL) and 104-2313-B-002-022-MY3 (to CCC), National Science Foundation Awards IOS-1121847 (to ACCW and DRGP), IOS-1354154 (to ACCW), and IOS-1257967 (to AHW).

\section{Competing interests}

The authors declare that they have no competing interests.

Received: 14 October 2015 Accepted: 22 December 2015

Published online: 11 January 2016

\section{References}

1. Shigenobu S, Wilson AC. Genomic revelations of a mutualism: the pea aphid and its obligate bacterial symbiont. Cell Mol Life Sci. 2011;68:1297-309.

2. Price DR, Feng H, Baker JD, Bavan S, Luetje CW, Wilson AC. Aphid amino acid transporter regulates glutamine supply to intracellular bacterial symbionts. Proc Natl Acad Sci USA. 2014;111:320-5.

3. Baumann P, Baumann L, Lai CY, Roubakhsh D, Moran NA, Clark MA. Genetics, physiology, and evolutionary relationships of the genus Buchneraintracellular symbionts of aphids. Annu Rev Microbiol. 1995;49:55-94. 
4. Wilkinson TL, Fukatsu T, Ishikawa H. Transmission of symbiotic bacteria Buchnera to parthenogenetic embryos in the aphid Acyrthosiphon pisum (Hemiptera: Aphidoidea). Arthropod Struct Dev. 2003;32:241-5.

5. Griffiths GW, Beck SD. Intracellular symbiotes of the pea aphid, Acyrthosiphon pisum. J Insect Physiol. 1973;19:75-84.

6. Buchner P. Endosymbiosis of animals with plant microorganisms. New York: Interscience; 1965.

7. Fukatsu T, Nikoh N, Kawai R, Koga R. The secondary endosymbiotic bacterium of the pea aphid Acyrthosiphon pisum (Insecta: Homoptera). Appl Environ Microbiol. 2000;66:2748-58.

8. Koga R, Meng XY, Tsuchida T, Fukatsu T. Cellular mechanism for selective vertical transmission of an obligate insect symbiont at the bacteriocyteembryo interface. Proc Natl Acad Sci USA. 2012;109:E1230-7.

9. Miura T, Braendle C, Shingleton A, Sisk G, Kambhampati S, Stern DL. A comparison of parthenogenetic and sexual embryogenesis of the pea aphid Acyrthosiphon pisum (Hemiptera: Aphidoidea). J Exp Zool B Mol Dev Evol. 2003;295:59-81.

10. Braendle C, Miura T, Bickel R, Shingleton AW, Kambhampati S, Stern DL. Developmental origin and evolution of bacteriocytes in the aphid-Buchnera symbiosis. PLoS Biol. 2003;1:E21.

11. Douglas AE. Phloem-sap feeding by animals: problems and solutions. J Exp Bot. 2006;57:747-54.

12. Price DR, Duncan RP, Shigenobu S, Wilson AC. Genome expansion and differential expression of amino acid transporters at the aphid/Buchnera symbiotic interface. Mol Biol Evol. 2011;28:3113-26.

13. Price DR, Wilson AC, Luetje CW. Proton-dependent glutamine uptake by aphid bacteriocyte amino acid transporter ApGLNT1. Biochim Biophys Acta. 2015;1848:2085-91.

14. Thwaites DT, Anderson CMH. The SLC36 family of proton-coupled amino acid transporters and their potential role in drug transport. Br J Pharmacol. 2011;164:1802-16.

15. Duncan RP, Husnik F, Van Leuven JT, Gilbert DG, McCutcheon JP, Wilson AC. Dynamic recruitment of amino acid transporters to the insect/symbiont interface. Mol Ecol. 2014;23:1608-23.

16. Dahan RA, Duncan RP, Wilson AC, Davalos LM. Amino acid transporter expansions associated with the evolution of obligate endosymbiosis in sap-feeding insects (Hemiptera: sternorrhyncha). BMC Evol Biol. 2015;15:52.

17. Wilson AC, Duncan RP. Signatures of host/symbiont genome coevolution in insect nutritional endosymbioses. Proc Natl Acad Sci USA. 2015;112:10255-61.

18. Matsuura Y, Kikuchi Y, Miura T, Fukatsu T. Ultrabithorax is essential for bacteriocyte development. Proc Natl Acad Sci USA. 2015;112:9376-81.

19. The International Aphid Genomics Consortium. Genome sequence of the pea aphid Acyrthosiphon pisum. PLoS Biol. 2010;8:e1000313.

20. Chang C-C, Lin GW, Cook CE, Horng SB, Lee HJ, Huang TY. Apvasa marks germ-cell migration in the parthenogenetic pea aphid Acyrthosiphon pisum (Hemiptera: Aphidoidea). Dev Genes Evol. 2007;217:275-87.

21. Kozak M. Structural features in eukaryotic mRNAs that modulate the initiation of translation. J Biol Chem. 1991;266:19867-70.

22. Rentsch D, Laloi M, Rouhara I, Schmelzer E, Delrot S, Frommer WB. NTR1 encodes a high-affinity oligopeptide transporter in Arabidopsis. FEBS Lett. 1995;370:264-8.

23. Fischer WN, Loo DDF, Koch W, Ludewig U, Boorer KJ, Tegeder M, et al. Low and high affinity amino acid $\mathrm{H}^{+}$-cotransporters for cellular import of neutral and charged amino acids. Plant J. 2002;29:717-31.

24. Gietz RD, Schiestl RH. Quick and easy yeast transformation using the LiAc/ SS carrier DNA/PEG method. Nat Protoc. 2007;2:35-7.

25. Sauer N, Stolz J. Expression of foreign proteins in yeast. In: Baldwin SA, editor. Membrane Transport. Oxford: Oxford University Press; 2000. p. 79-105.

26. Laemmli UK. Cleavage of structural proteins during assembly of head of bacteriophage T4. Nature. 1970;227:680-5.

27. Chang C-C, Huang TY, Shih CL, Lin GW, Chang TP, Chiu H, et al. Wholemount identification of gene transcripts in aphids: protocols and evaluation of probe accessibility. Arch Insect Biochem Physiol. 2008;68:186-96.

28. Chang C-C, Lee WC, Cook CE, Lin GW, Chang T. Germ-plasm specification and germline development in the parthenogenetic pea aphid Acyrthosiphon pisum: Vasa and Nanos as markers. Int J Dev Biol. 2006;50:413-21.

29. Lin GW, Cook CE, Miura T, Chang C-C. Posterior localization of ApVas1 positions the preformed germ plasm in the sexual oviparous pea aphid Acyrthosiphon pisum. EvoDevo. 2014;5:18.
30. Shigenobu S, Stern DL. Aphids evolved novel secreted proteins for symbiosis with bacterial endosymbiont. Proc Biol Sci. 2013;280:20121952.

31. Hansen AK, Degnan PH. Widespread expression of conserved small RNAs in small symbiont genomes. ISME J. 2014;8:2490-502.

32. Nakabachi A, Ishida K, Hongoh Y, Ohkuma M, Miyagishima SY. Aphid gene of bacterial origin encodes a protein transported to an obligate endosymbiont. Cur Biol. 2014;24:R640-1.

33. Brough CN, Dixon AFG. Follicular sheath (Ovarian Sheath) structure in Virginoparae of the vetch aphid, Megoura viciae buckton (Homoptera: Aphididae). Int J Insect Morphol Embryol. 1989;18:217-26.

34. Hagan HR. Pseudoplacental Viviparity-Corrodentia, Hemiptera (Aphididae). In: Hagan HR, editor. Embryology of the viviparous insects. New York: Roland Press; 1951. p. 347-92.

35. Telfer WH. Development and physiology of the oocyte-nurse cell syncytium. Adv Insect Physiol. 1975;11:223-321.

36. Johannsen OA, Butt FH. HOMOPTERA, APHIDIDAE. In: Johannsen OA, Butt $\mathrm{FH}$, editors. Embryology of Insects and Myriapods. The developmental history of insects, centipedes, and millepedes from egg desposition to hatching. New York: McGraw-Hill Book company, Inc.; 1941. pp. 259-68.

37. Blackman RL. Reproduction, cytogenetics and development. In: Minks AK, Harrewijn P, editors. Aphids: their biology, natural enemies and control, vol. A. Amsterdam: Elsevier; 1987. p. 163-95.

38. Bermingham J, Wilkinson TL. Embryo nutrition in parthenogenetic viviparous aphids. Physiol Entomol. 2009;34:103-9.

39. Blackman RL. Early development of parthenogenetic egg in 3 species of aphids (Homoptera Aphididae). Int J Insect Morphol. 1978;7:33-44.

40. Buning J. Morphology, ultrastructure, and germ cell cluster formation in ovarioles of aphids. J Morphol. 1985;186:209-21.

41. Ogmundsdottir MH, Heublein S, Kazi S, Reynolds B, Visvalingam SM, Shaw MK, et al. Proton-assisted amino acid transporter PAT1 complexes with Rag GTPases and activates TORC1 on late endosomal and lysosomal membranes. PLoS One. 2012;7:e36616.

42. Chantranupong $\mathrm{L}$, Wolfson $\mathrm{RL}$, Sabatini DM. Nutrient-sensing mechanisms across evolution. Cell. 2015;161:67-83.

43. Taylor PM. Role of amino acid transporters in amino acid sensing. Am J Clin Nutr. 2014;99:223S-30S

44. Goberdhan DC, Meredith D, Boyd CA, Wilson C. PAT-related amino acid transporters regulate growth via a novel mechanism that does not require bulk transport of amino acids. Development. 2005;132:2365-75.

45. Goberdhan DC. Intracellular amino acid sensing and mTORC1-regulated growth: new ways to block an old target? Curr Opin Investig Drugs. 2010;11:1360-7.

46. Bermingham J, Rabatel A, Calevro F, Vinuelas J, Febvay G, Charles H, et al. Impact of host developmental age on the transcriptome of the symbiotic bacterium Buchnera aphidicola in the pea aphid (Acyrthosiphon pisum). Appl Environ Microbiol. 2009;75:7294-7.

47. Rabatel A, Febvay G, Gaget K, Duport G, Baa-Puyoulet P, Sapountzis P, et al. Tyrosine pathway regulation is host-mediated in the pea aphid symbiosis during late embryonic and early larval development. BMC Genom. 2013;14:235.

48. Putignani L, Del Chierico F, Petrucca A, Vernocchi P, Dallapiccola B. The human gut microbiota: a dynamic interplay with the host from birth to senescence settled during childhood. Pediatr Res. 2014;76:2-10.

49. McFall-Ngai MJ. The importance of microbes in animal development: lessons from the squid-vibrio symbiosis. Annu Rev Microbiol. 2014;68:177-94.

50. Graham ER, McKie-Krisberg ZM, Sanders RW. Photosynthetic carbon from algal symbionts peaks during the latter stages of embryonic development in the salamander Ambystoma maculatum. BMC Res Notes. 2014;7:764.

51. Hansen AK, Moran NA. Aphid genome expression reveals host-symbiont cooperation in the production of amino acids. Proc Natl Acad Sci USA. 2011;108:2849-54.

52. Ward A, Sanderson NM, O'Reilly J, Rutherford NG, Poolman B, Henderson PJF. The amplified expression, identification, purification, assay, and properties of hexahistidine-tagged bacterial membrane transport proteins. In: Baldwin SA, editor. membrane transport. Oxford: Oxford University Press; 2000. p. 141-66. 